\title{
Mechanical Integrity of Steel Discs with Corrosion Pits
}

\author{
Jose Urbano ${ }^{1, *}$, Andrej Golowin ${ }^{2}$, Stefan Löhnert ${ }^{3}$ and Dieter Bestle ${ }^{1}$ \\ ${ }^{1}$ Chair of Engineering Mechanics and Vehicle Dynamics, Brandenburg University of Technology, Siemens-Halske-Ring 14, \\ 03046, Cottbus, Germany \\ ${ }^{2}$ Rolls-Royce Deutschland Ltd \& Co KG, Eschenweg 11, 15827 Blankenfelde-Mahlow, Germany \\ ${ }^{3}$ Institute of Continuum Mechanics, Leibniz University Hannover, Appelstraße 11, 30167 Hannover, Germany
}

\begin{abstract}
Currently, prediction of crack initiation by corrosion pits is only possible by assuming regular geometrical shapes, such as semi-spheres or semi-ellipsoids. Moreover, typical fatigue life diagrams associate the crack initiation life with geometrical features, such as pit depth or aspect ratio, often leading to unsatisfactory correlations due to high pit shape variability and data scatter. In the context of blade-disc fixation in aero engine turbines, this limitation translates into highly conservative life estimations. Therefore, a new crack initiation predictor is formulated based on experimental testing and numerical analysis of 28 artificial corrosion pits. A low-cycle fatigue test campaign is conducted using three-point bending test specimens to simulate maximum takeoff operation conditions of the aero engine and the associated loading of the blade root designed as firtree. An artificial pit is located at the critical point of each test specimen, respectively. The prediction criterion is based on finite element analysis and is formulated as the lowest plastic strain of a plastic region with a certain volume in the corrosion pit. This reference volume is varied until an optimum correlation with experimental crack initiation life is obtained. The criterion shows a superior correlation with crack initiation life compared to pure geometrical parameters such as pit depth.
\end{abstract}

\section{Introduction}

Corrosion of critical parts is one of the key cost drivers in aerospace industry. Corrosion problems typically appear in the mature fleet and are considered as most difficult and expensive due to the potential size of the affected fleet and variety of factors influencing life prediction and fleet management.

In the design of steel discs for high-pressure turbines (HPT), corrosion criticality is evaluated by estimating the number of cycles required to initiate a crack by a corrosion pit (crack initiation life), and the number of cycles required for this crack to propagate and cause macroscopic fracture (crack propagation life). The crack propagation life is typically obtained from a linear elastic fracture analysis with an initial crack size equal to the depth of the corrosion pit [1-4]. The crack initiation life may also be estimated by crack propagation analysis until a specific engineering crack $[5,6]$, crack growth rate or stress intensity factor is reached $[1-4,7,8]$. To overcome the high conservatism in such a crack initiation life calculation, current research is conducted on two major fronts.

The first focuses on formulating improved analytical models for estimating stress intensity factors using linear elastic fracture mechanics, see [8-10]. Recent efforts have been devoted to enhance the pit model by considering more specific shape factors, accurate stress concentration factors using finite element simulations, and multi-step crack growth hypotheses $[3,4]$. One of the

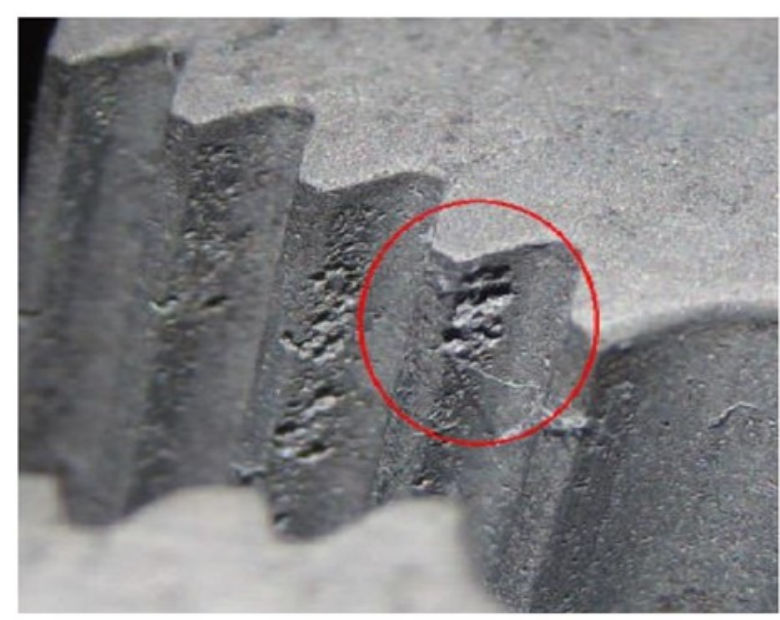

Fig. 1. Corrosion pits at the firtree joint of an HPT disc.

major drawbacks of these approaches relies on the approximation of the pit shape as hemispherical or semielliptical. Although this is a necessary assumption for the development of analytical solutions, in reality the corrosion pit shape is rather irregular (Fig. 1), and it must be taken into account since it may have important implications on local strain concentrations.

The second research front deals with an improved micromechanical description of the crack initiation mechanisms using the continuum damage approach $[11,12]$. Damage models offer an analytical description of the mechanical interaction between pit growth and

\footnotetext{
* Corresponding author: urbanoca@b-tu.de
} 
damage laws. Improved technologies such as accelerated 3D microstructure characterization may facilitate the applicability of such models in the future [13], however, due to the increased amount of model parameters, the industrial application of such approaches is still limited.

Instead of developing an analytical model for crack growth based on geometric simplifications, the present paper aims at formulating a crack initiation predictor which considers the real pit geometry and individual load level. The method is developed to predict the crack initiation life of a corroded high-pressure turbine disc of an aero engine. A high-pressure turbine disc is prone to corrosion pitting at the firtree joint, which is the location of highest mechanical loading (Fig. 1). Therefore, the mechanical integrity of the turbine disc may be evaluated by estimating the required number of cycles for crack initiation by a corrosion pit located at the firtree.

In practical terms, a crack initiation predictor is a criterion which exhibits a useful correlation with real crack initiation life. To formulate this predictor, an experimental test campaign with 28 simplified test specimens representing the disc firtree is carried out. Each test specimen contains an artificially created corrosion pit (hereafter referred as artificial pit), which imitates the shape of corrosion pits found in used HPT discs (hereafter referred as ex-service pit). From this experimental test campaign, crack initiation lives $N_{I, i}$ of all artificial pits are obtained. The surface scans of the artificial pits are used in a finite element analysis, and from the individual simulation results a predictor $q_{i}$ is formulated for each artificial pit $i$. The goal is to maximize the correlation of the data set

$$
\left(N_{I, i}, N_{I}\left(q_{i}\right)\right), \quad i=1, \ldots, 28,
$$

between the experimentally found crack initiation life $N_{I, i}$ and the estimate $N_{I}\left(q_{i}\right)$ based on the numerical predictor $q_{i}$.

The paper is organized as follows: after describing the experimental test campaign, a finite element model of the test specimen will be discussed. A crack initiation predictor will be formulated using the representativevolume method, and validated with the data set (1). Finally, the found correlation will be applied to estimate the crack initiation life of ex-service pits.

\section{Experimental test campaign}

Since it is not possible to properly load and test corrosion pits on HPT discs directly, a test campaign based on a simplified mechanical model of the disc firtree was developed and a three-point bending test was chosen, see Fig. 2. The geometry of the specimen is designed as a rectangular plate with a $\mathrm{V}$-notch such that the angle and depth of the V-notch correspond to the firtree lobe, Fig. 3. A population of 28 test specimens was manufactured using ex-service HPT discs for an ideal representation of material properties.

Each manufactured test specimen was sent to the National Physical Laboratory (NPL) at Teddington, UK, for artificial pit application. A corrosion experiment was

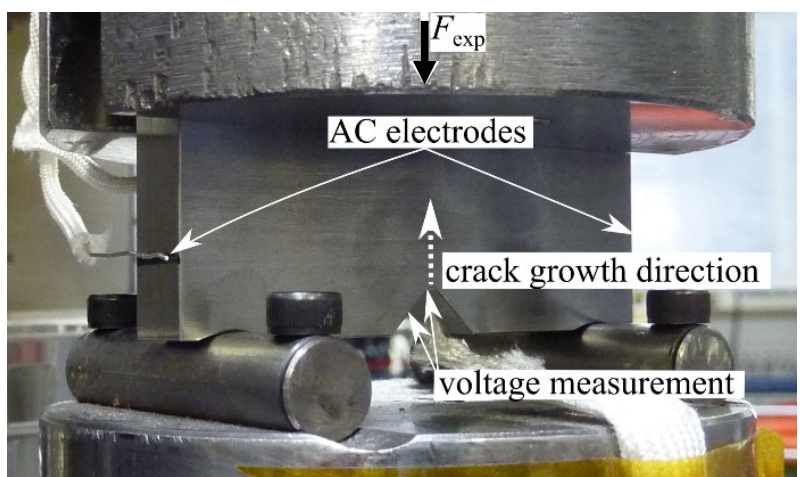

Fig. 2. Instrumentation of the test specimen.

designed to produce artificial pits with shapes and dimensions similar to those observed in ex-service discs. Each artificial pit is created using a droplet of saline solution on an electrified test specimen. Since the highest stress occurs at the bottom of the V-notch, it was decided to incubate the artificial pits at this location, see Fig. 3. The diameter of the droplet, the electrical current, and the exposure time where controlled to achieve the requested pit depth, as well as desired major and minor pit diameters. The 3D images of the artificial pits were compared to those taken from ex-service pits to ensure representativeness.
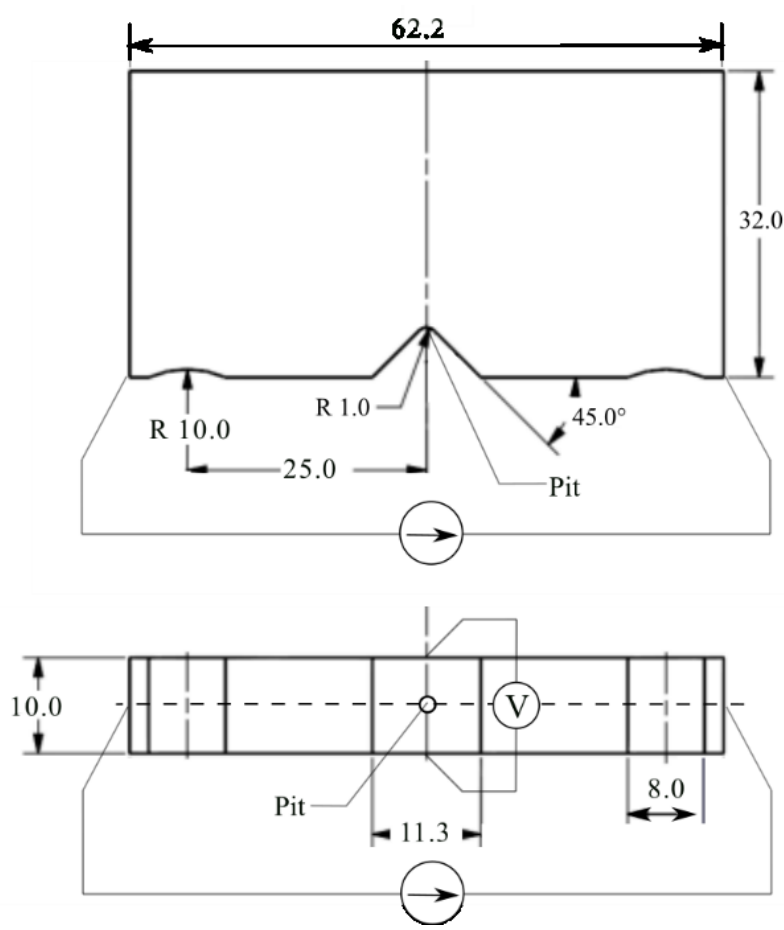

Fig. 3. Test specimen for three-point bending test (dimensions given in $\mathrm{mm}$ ).

The pitted specimens were sent back to the RollsRoyce Mechanical Test Operations Centre (MTOC) at Dahlewitz, Germany, for low-cycle fatigue testing. A single load cycle is defined by four loading stages: $1 \mathrm{~s}$ linearly increasing loading ramp to a maximum load $F_{\text {exp }}$, $1 \mathrm{~s}$ constant loading at $F_{\text {exp }}, 1 \mathrm{~s}$ linearly decreasing loading ramp until $10 \%$ of $F_{\text {exp }}$, and $1 \mathrm{~s}$ constant loading at $10 \%$ of $F_{\text {exp }}$. The test cycles were repeated until failure by fracture and counted by $N_{T}$. 
The maximum load $F_{\text {exp }}$ is designed to achieve a desired stress field at the V-notch. It is based on the nominal load $F_{\text {nom }}$ which produces the same stress field at the $\mathrm{V}$-notch as the one in the firtree lobe during maximum takeoff. This loading is considered as the maximum loading during the complete flight cycle. Specimens with different pit depths were assigned to one of the four load levels

$$
F_{\text {exp }}=\alpha \mathrm{F}_{\text {nom }}
$$

where $\alpha \in\{95 \%, 100 \%, 105 \%, 110 \%\}$,

such that each of seven pit depth magnitudes was combined with each of the four load levels. The specimen is supported by two rollers on the lower side, see Fig. 2, and the contact between the loading piston and the upper side of the test specimen is a similar internal roller inside the piston with the same radius as the support rollers (not visible in Fig. 2, but see model in Fig. 6a).

The artificial pit intensives the nominal stress at the $\mathrm{V}$-notch. Since this location experiences the highest bending stress, this is where crack initiation is expected. The crack growth follows the path of highest stress gradient, which in this case is perpendicular to the bending direction, see Fig. 2. The crack growth is estimated by inducing an alternating electric current across the test specimen and measuring the change of the electric potential $v$ in the $\mathrm{V}$-notch (see Fig. 2 for instrumentation location). A typical behavior of voltage $v$ vs. number of test cycles $N_{T}$ is shown in Fig. 4.

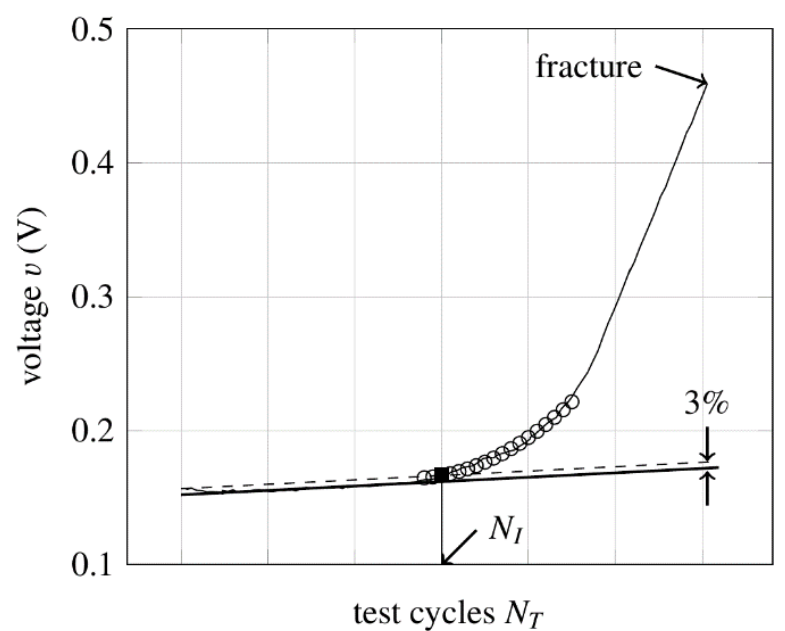

Fig. 4. Voltage at the test specimen vs. number of test cycles: raw signal (-), linear fit (-), 3\% offset line (--), polynomial fit (०), and point of intersection ( $(\mathbf{})$.

In the beginning, the increase is linear and rather slow. Here, a linear crack growth may be assumed, and thus a direct proportional relation between measured voltage and crack length. After a specific amount of cycles, the crack growth is significantly accelerated. The amount of cycles up to this transition point is defined as the number of cycles for crack initiation $N_{I}$ (also crack initiation life). The remaining cycles until specimen failure refer to crack propagation.

The identification of the cycles to crack initiation $N_{I}$ is obtained by the following procedure: two sets of experimental data points are selected. The first set is taken at the initial stage of crack growth and a linear curve is fitted to this data set. Additionally, a second line with a $3 \%$ offset is defined. The second set of points is taken from the middle region of the test curve and a third-order polynomial is fitted to this data set. The point of intersection between the polynomial approximation and the $3 \%$ offset line is defined as number of cycles $N_{I}$ to crack initiation. The range of the two data sets is based on experience from previous crack initiation measurements.

Traditionally, the crack initiation life is correlated with geometrical predictors, such as pit depth or aspect ratio. Such a correlation between the artificial pit depth $D$ and the experimentally found value $N_{I}$ for crack initiation for all 28 tested specimens is shown in Fig. 5. Despite the high scatter in the data, some relationship between crack initiation life and load level may be deduced: as the load level increases, crack initiation life becomes shorter. Further, the relevance of the pit depth seems to be more influential at lower load levels. For example, at 95\% load level an increase in pit depth is generally associated with some shortening of crack initiation life, however, pit depth has almost no influence at $110 \%$ load level. Thus, this correlation picture of crack initiation life with pit depth has obvious limitations. First, a clear distinction of different load levels should be performed which, however, would decrease the population size and thus statistical significance. Furthermore, it must be considered that the pit shapes are extremely variable and irregular. Therefore, different crack initiation lives may be expected from different pits with same pit depth. In the following, a crack initiation predictor will be developed to overcome such limitations.

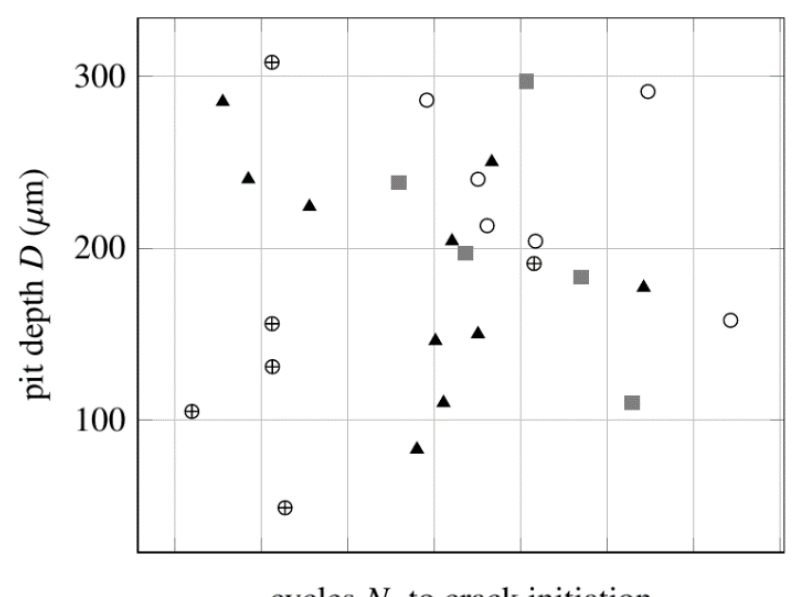

cycles $N_{I}$ to crack initiation

Fig. 5. Pit depth vs. crack initiation life for different load

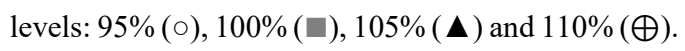

\section{Finite element model of test specimen}

Crack nucleation involves an interaction of elastic stress and plastic strain concentrations $[3,6,14]$. Thus, a crack predictor should consider the intrinsic mechanical loading at the pit to provide a more satisfactory correlation with crack initiation. Furthermore, a representative measure of the plastic strain concentration at the pit would make the desired predictor independent of the pit geometry. For 
these reasons, the goal is to formulate a crack initiation predictor based on the actual plastic strain concentration at the pit, which will be calculated from a finite element analysis.

A structural simulation of a corrosion pit using real surface measurements is possible by the following two steps: firstly, a geometry processing workflow in Siemens NX to consider the measured pit surface in the specimen CAD geometry; secondly, a plastic analysis in Abaqus CAE to obtain an accurate representation of the stress and strain field at the pit.

The geometry processing workflow prepares the surface scan to be applied to the model geometry. Each artificial pit was scanned with a high-precision laserscanning microscope at NPL, and the surface scan was then processed in Siemens NX to result in an applicable CAD geometry. First, the surface scan was trimmed to the internal pit surface. Then, a smoothing was performed by fitting a B-spline surface to the trimmed surface scan, which facilitates the subsequent finite element meshing. Finally, the surface was positioned in a CAD geometry of the test specimen, and the material above the pit surface was removed using an extrusion procedure. This surface processing is similar to the one reported in [3]. It should be emphasized that the transition from the pit surface to the outer specimen surface should be kept as smooth as possible to avoid meshing errors. Fortunately, most of the high plastic strain regions are located at the inside of the pit, as it will be demonstrated later. Therefore, the relevant regions are barely affected by this rim smoothing.
The second step starts with the preparation of a finite element model, where the goal is to obtain a precise representation of the strain field at the pit surface. For this purpose, a global model is used for a coarse finite element analysis of the whole test specimen (Fig. 6a), and the pit region is then refined using two sequential submodels (Fig. 6b-c). The global model determines displacements of the test specimen considering load and fixation boundary conditions, but not the pit. Then, a first submodel transfers the obtained nodal displacements to the boundaries of a section of the specimen near the pit and calculates displacement with a refined mesh, where the pit is still not considered (Fig. 6b). Only the second sectional submodel in Fig. 6c has a sufficiently high mesh refinement to include the corrosion pit for obtaining the desired stress and strain information of the pit region. Of course, the section walls for the submodels must be positioned at locations where the strain field is not affected by the missing pit.

The global model in Fig. 6a considers the complete test assembly composed of test specimen, the two support cylinders, and an upper half-cylinder transmitting the piston load. The lower half of the support rollers is completely constrained, whereas the upper half has a friction contact with the specimen surface (friction coefficient $\mu=0.4$ ). The contact between the upper half cylinder and the test specimen is simulated as a non-slip contact, and the uniform pressure on the upper cylinder matches the experimental load $F_{\text {exp }}$.

(b)

(c)

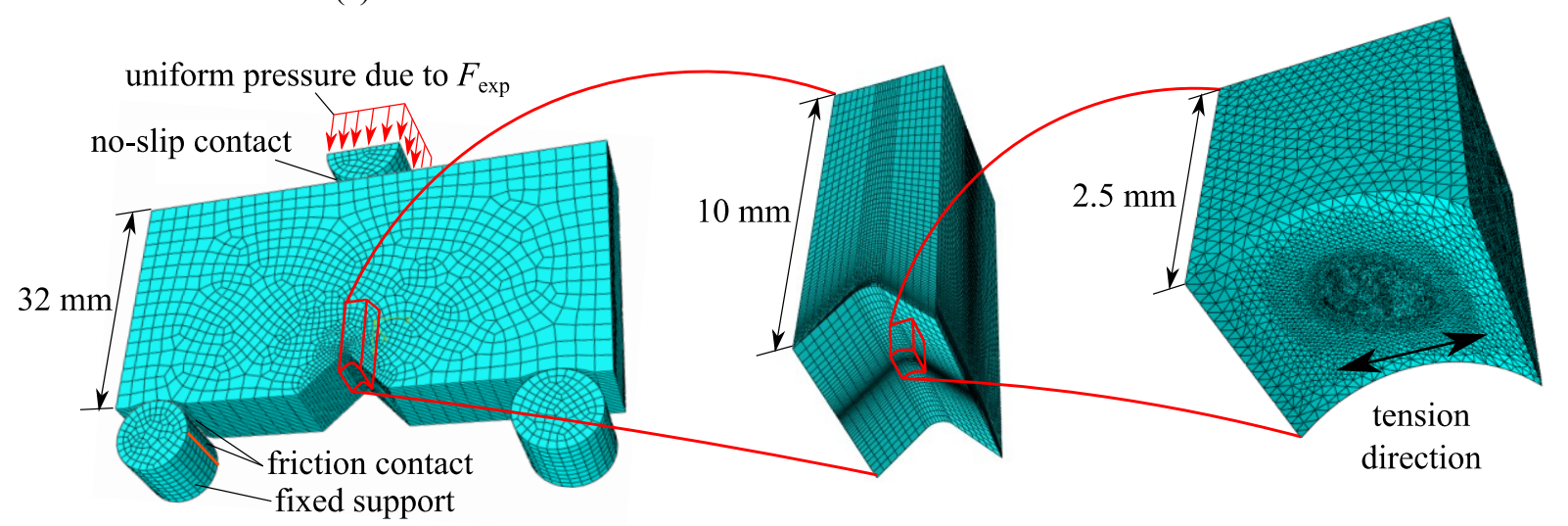

Fig. 6. Meshing of specimen-roller assembly: (a) global model, (b) first and (c) second submodel. (a)

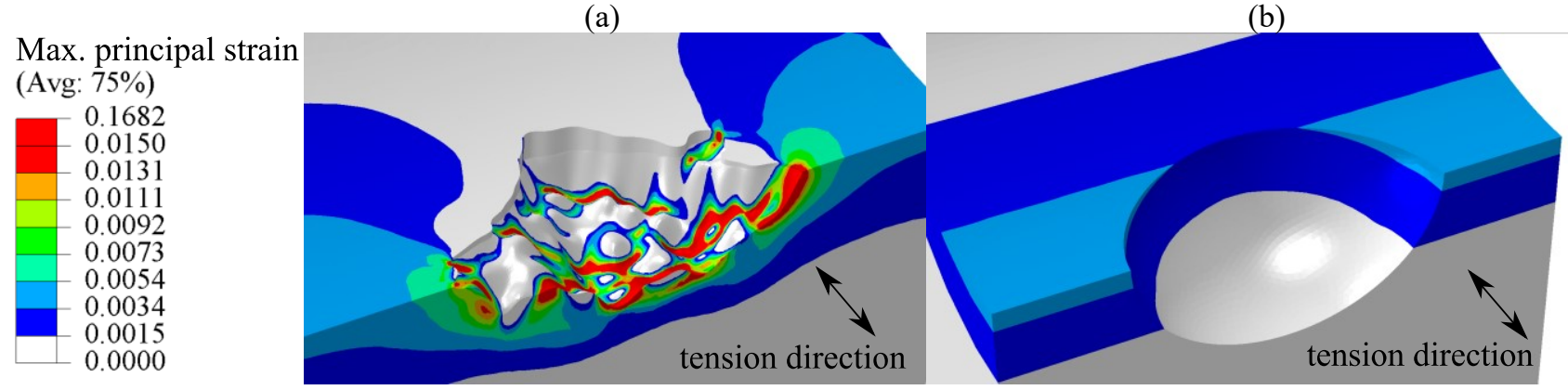

Fig. 7. Maximum plastic strain for an (a) ex-service and (b) idealized pit. 
The static plastic analyses of the three models are conducted with Abaqus CAE. An isotropic plastic hardening material model based on the von Mises yield criterion is utilized for the test specimen. The stress-strain relationship at room temperature for the disc material (FV535 steel) is taken from [15]. Although the experiment involves cyclic loading, the simulation considers only the first loading ramp. This is consistent with the isotropic plastic hardening assumption, since no further incremental strain should be expected by simulating additional cycles.

The global model is meshed with second order hexahedral elements. The first submodel refines the region about the V-notch also with second order hexahedral elements. Finally, the second submodel is meshed using second order tetrahedral elements to provide the required geometric flexibility for modelling the pit surface. The average element size in the second submodel is $0.015 \mathrm{~mm}$, and the mesh consists of approximately 180000 elements.

The geometry processing and the finite element analysis are applied to all 28 test specimens of the test campaign in Section 2 using individually measured pit surfaces and individual load levels to obtain the plastic strain values. This procedure is also suitable in Section 5, to simulate ex-service pits located in a HPT firtree.

In order to see the need of taking into account the real pit geometry, plastic strains are computed for one of the ex-service pits and compared to those of an idealized pit, which is defined as the smallest half-ellipsoid that completely encloses the ex-service pit, see Fig. 7 . The latter is a classical simplification used in finite element modelling of corrosion pits, see $[9,16]$. The sectional view at the middle plane of the pit reveals that the ex-service pit has a totally different strain pattern than the idealized pit, although both have identical boundary conditions. Critical areas in Fig. 7a with high plastic strain occur mainly due to surface irregularities of the pit, especially at surface peaks and valleys. These high-strain regions tend to group into longitudinal bands perpendicular to the direction of tension, which has also been observed on previous finite element analyses of corrosion pits $[3,9,16]$. Regions with highest plastic strains most likely represent potential crack nucleation sites [3]. Therefore, beside plastic strain values, an additional outcome of the shown finite element analysis is the identification of potential crack nucleation locations.

\section{Crack initiation prediction with the representative-volume method}

Although the plastic strain provides insight in the location and severity of crack nucleation sites, a summarizing criterion for comparison of different pits is still absent. Therefore, the goal of the present section is to define a strain measure which is representative for the whole pit. In the following, the plastic strain will be associated with a characteristic volume, which is why this method will be called representative-volume method. The idea of the method is to identify the volume of the most critical plastic strain regions, such that its reference volume
$V_{\text {ref }}$ and associated plastic strain $\varepsilon_{\text {ref }}^{P}$ may be used for crack initiation life predictions which maximize the correlation with experimentally found results.

Let us define the index subset $\mathbb{D}$ as the collection of element indexes $e$ whose maximum principal plastic strain $\varepsilon_{\max , e}^{P}$ exceeds a prescribed reference plastic strain $\varepsilon_{\text {ref }}^{P}$, i.e.,

$$
\mathbb{D}=\left\{e: \varepsilon_{\text {max }, e}^{P} \geq \varepsilon_{\text {ref }}^{P}\right\} .
$$

The size $|\mathbb{D}|$ of this subset depends on the value of $\varepsilon_{\text {ref }}^{P}$, where a higher reference plastic strain will lead to a smaller subset $\mathbb{D}$. The sum of volumes $V_{e}$ of all elements belonging to $\mathbb{D}$ is defined as the reference volume

$$
V_{\mathrm{ref}}=\sum_{e \in \mathbb{D}} V_{e}
$$

The relationship $V_{\text {ref }}=V_{\text {ref }}\left(\varepsilon_{\text {ref }}^{P}\right)$ between reference volume and reference plastic strain is individual to every pit and may be graphically represented in a volumetric accumulation diagram as shown in Fig. 8. This diagram reveals the volumetric distribution of plastic strains in the pit, and may be obtained by the following procedure:

1. For each element $e$ of the second submodel in Fig. 6c, compute the maximum principal plastic strain $\varepsilon_{\max , e}^{P}$ at the element centroid by finite element analysis.

2. Sort the values of the maximum plastic strain in ascending order to get an element index set

$$
\begin{aligned}
\mathbb{E}= & \left\{e_{1}, \cdots, e_{j}, \cdots, e_{N_{E}}\right\} \\
& \text { where } \varepsilon_{\text {max }, e_{j+1}}^{P} \geq \varepsilon_{\text {max }, e_{j}}^{P},
\end{aligned}
$$

and $N_{E}$ corresponds to the number of elements in the second submodel.

3. Calculate the reference volumes $V_{\text {ref }, j}$ associated with occurring reference plastic strains $\varepsilon_{\mathrm{ref}, j}^{P}$ recursively as

$$
\begin{aligned}
\varepsilon_{\mathrm{ref}, j} & =\varepsilon_{\mathrm{max}, e_{j}}^{P} \\
V_{\mathrm{ref}, j} & =V_{\mathrm{ref}, j+1}+V_{e_{j}} \\
& \text { where } e_{j} \in \mathbb{E} \\
& V_{\mathrm{ref}, N_{E}}=V_{e_{N_{E}}} \\
& \varepsilon_{\mathrm{ref}, N_{E}}=\varepsilon_{\max , e_{N_{E}}}^{P} \\
& j=N_{E}-1, N_{E}-2, \ldots, 1 .
\end{aligned}
$$

The results (6) and (7) define the volumetric accumulation diagram for a specific pit as

$$
V_{\mathrm{ref}, j}=V_{\mathrm{ref}}\left(\varepsilon_{\mathrm{ref}, j}^{P}\right), j=1, \cdots, N_{E} .
$$

Additionally, a reference length $L_{\text {ref }}$ associated with the reference volume $V_{\text {ref }}$ may be introduced as 


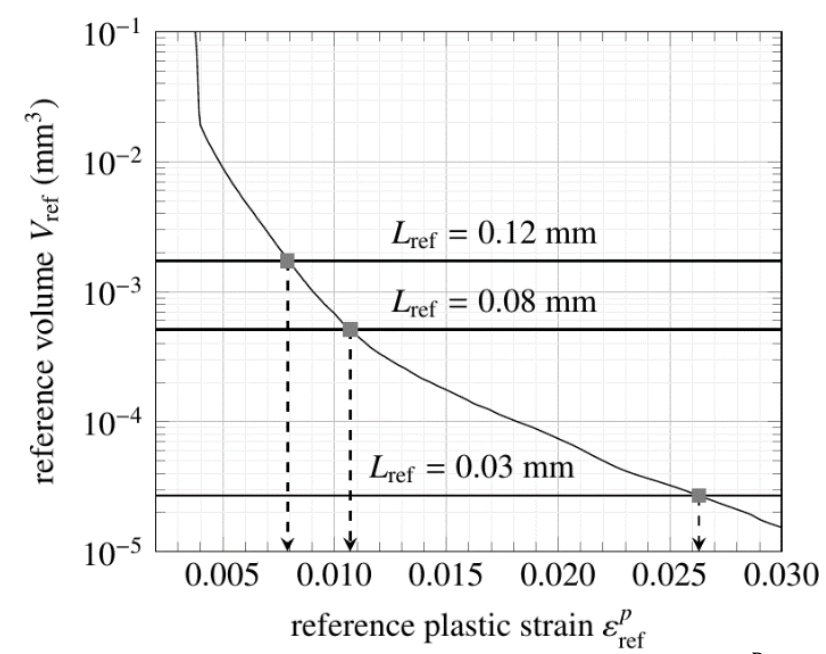

Fig. 8. Reference volume $V_{\text {ref }}$ vs. reference plastic strain $\varepsilon_{\text {ref }}^{P}$ for a specific artificial pit.

$$
L_{\mathrm{ref}}=\sqrt[3]{V_{\mathrm{ref}}}
$$

The volumetric accumulation diagram in Fig. 8 associates a plastic strain $\varepsilon_{\text {ref }}^{P}$ with the size of a plastic region $V_{\text {ref. For instance, the reference lengths }}$ $L_{\text {ref }}=0.12 \mathrm{~mm}, 0.08 \mathrm{~mm}$, and $0.03 \mathrm{~mm}$ are associated with the plastic strains $\varepsilon_{\text {ref }}^{P}=0.0079,0.0107$, and 0.02629. Obviously, larger element volumes are associated with lower plastic reference strains, Fig. 9.

Based on the assumption that high plastic strains will increase the probability of crack initiation, and thus shorten crack initiation life, we may now reproduce Fig 8 for all 28 artificial pits of the test campaign in Section 2. This is done by extracting plastic reference strains $\varepsilon_{\text {ref }, i}^{P}$ for prescribed values of $L_{\text {ref }}$ and correlating them to the experimentally obtained crack initiation lives $N_{I, i}$, see Fig. 10. Obviously there is a strong correlation between these two quantities, which is why

$$
q:=\varepsilon_{\text {ref }}^{P}\left(V_{\text {ref }}\right) \text { or } \quad q:=\varepsilon_{\text {ref }}^{P}\left(L_{\text {ref }}\right)
$$

may act as a predictor for estimating the crack initiation life $N_{I}$ by

$$
\ln N_{I}=-m \ln q+a
$$

An optimal choice for the slope $(-m)$ and $y$-intercept $a$ may be found by a least-square fitting of the data $\left(q_{i}=\varepsilon_{\mathrm{ref}, i}^{P}, N_{I, i}\right), i=1, \ldots, 28$, to fulfil Eq. (11), i.e.,

$$
\min _{m, a} \sum_{i=1}^{28}\left(\ln N_{I, i}+m \ln q_{i}-a\right)^{2},
$$

see solid lines in Fig. 10.

The fact that a rather general reference strain $\varepsilon_{\text {ref }}^{P}$ corresponding to a reference volume $V_{\text {ref }}$ of material act as a reliable predictor in a wide range (compare Figures $10 \mathrm{a}-\mathrm{c}$ with $L_{\text {ref }}$ ranging from $0.03 \mathrm{~mm}$ to $0.12 \mathrm{~mm}$ ) indicates, that not only the singular point of maximum plastic strain $\varepsilon_{\max }^{P}$ is responsible for crack initiation. Instead, a whole region with plastic strains above some lower threshold $\varepsilon_{\mathrm{ref}}^{P}<\varepsilon_{\max }^{P}$ may be exposed to microcracks which then coalesce to a macroscopic crack determining the crack initiation life $N_{I}$.

The quality of the data fitting (12) may be quantified by the correlation coefficient

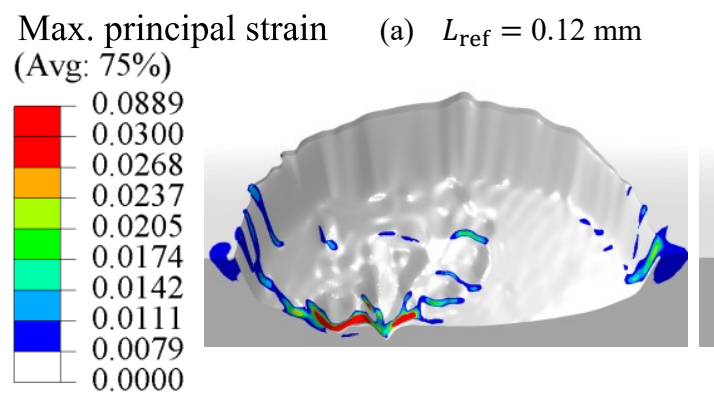

(b) $L_{\text {ref }}=0.08 \mathrm{~mm}$

(c) $L_{\mathrm{ref}}=0.03 \mathrm{~mm}$

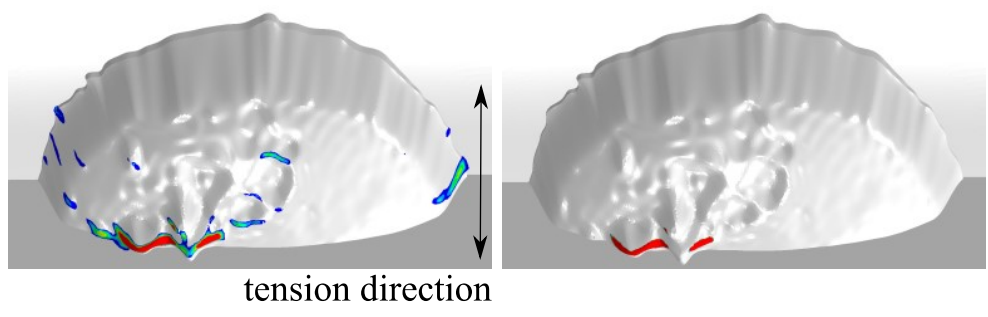

Fig. 9. Elements with maximum principal strain $\varepsilon_{\max }^{P}$ above (a) $\varepsilon_{\mathrm{ref}}^{P}=0.0079$, (b) $\varepsilon_{\mathrm{ref}}^{P}=0.0107$, and (c) $\varepsilon_{\mathrm{ref}}^{P}=0.02629$.

(a) $L_{\text {ref }}=0.12 \mathrm{~mm}$



(b) $L_{\text {ref }}=0.08 \mathrm{~mm}$

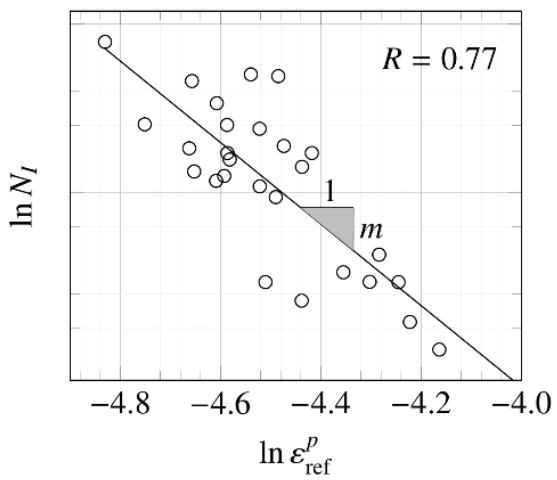

(c) $L_{\mathrm{ref}}=0.03 \mathrm{~mm}$

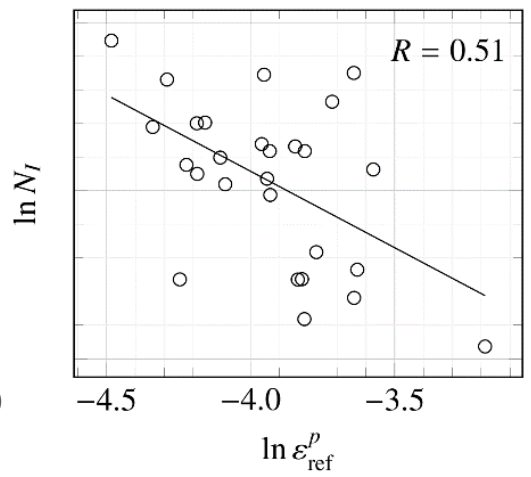

Fig. 10. Cycles to initiation life $\ln \left(N_{I}\right)$ for all artificial pits $p_{i}$ vs. reference plastic strain $\varepsilon_{\text {ref }}^{P}$ associated with various representative lengths. 


$$
\begin{gathered}
R=\frac{n \sum N_{I, i} \widetilde{N}_{I, i}-\left(\sum N_{I, i}\right)\left(\sum \widetilde{N}_{I, i}\right)}{\sqrt{n \sum N_{I, i}^{2}-\left(\sum N_{I, i}\right)^{2}} \sqrt{n \sum \widetilde{N}_{I, i}^{2}-\left(\sum \widetilde{N}_{I, i}\right)^{2}}} \\
\text { where } \widetilde{N}_{I, i}=\exp \left(-m \ln q_{i}+a\right) .
\end{gathered}
$$

The coefficient $R$ is a statistical measure for the deviation between the experimentally obtained $\left(N_{I, i}\right)$ and the predicted $\left(\widetilde{N}_{I, i}\right)$ cycles to crack initiation. The coefficient delivers values in the range $-1 \leq R \leq 1$, where 1 indicates a perfect match.

According to Fig. 10, the strength of correlation depends on the reference length. A larger reference length $L_{\text {ref }}=0.12 \mathrm{~mm}$ supposes that rather low plastic strain values may influence crack initiation life, Fig. 10a, which, however, is inadequate. A small reference length $L_{\text {ref }}=0.03 \mathrm{~mm}$ corresponding to a small reference volume assesses only elements with the highest plastic strain peaks in the corrosion pits, Fig. 10c. The corresponding correlation is also unsatisfactory, since these highest strain values usually occur from mesh irregularities or sharp edges. Therefore, the representative length producing the best correlation has to be chosen carefully.

In order to identify the optimum reference length (or volume), several reference lengths have been fixed and the corresponding correlation coefficient for the artificial pits has been calculated, see Fig 11, where the grey markers correspond to the results in Fig.10. Obviously, the reference length $L_{\text {ref }}=0.08 \mathrm{~mm}$ delivers the highest value of the correlation coefficient, and thus the best correlation (which can also be seen in Fig. 10b). The optimal values of representative length, volume and plastic strain are denoted as $L_{R}, V_{R}$ and $\varepsilon_{R}$, respectively.

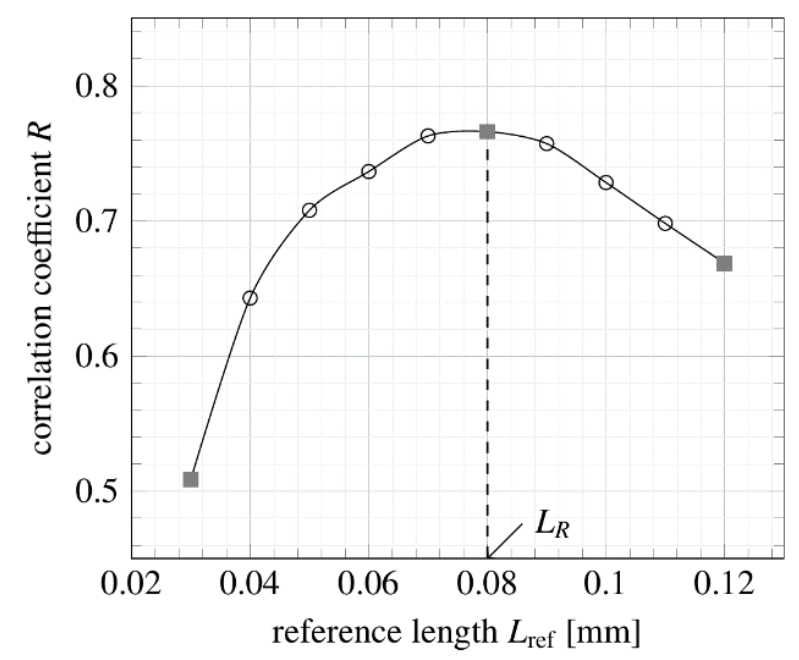

Fig. 11. Correlation coefficient $R$ vs. characteristic length $L_{\text {ref }}$ for tested specimens for test population.

A striking finding for application is that the optimum reference strain is rather robust against mesh refinement. Fig. 12 shows volumetric accumulation diagrams for one of the artificial pits (different from the artificial pit used in Fig. 8) using different mesh refinements. As expected, a higher mesh refinement may lead to a more precise description of regions with high plastic strain, (i.e., regions with low reference length $L_{\text {ref }}<0.03 \mathrm{~mm}$ ), however, for the optimum reference length $L_{R}=0.08 \mathrm{~mm}$ nearly the same value $\varepsilon_{R}$ is obtained from all finite element analyses. Therefore, an expensive mesh refinement of the pit surface is unnecessary for getting an optimal correlation with crack initiation life.

Throughout this work, the plastic strain (10) is used as a predictor for crack initiation life. The same procedure may also be based on stress values instead. However, since plastic strain is more sensitive than stress to load increments, a better correlation was observed by using the plastic strain as a crack initiation predictor.

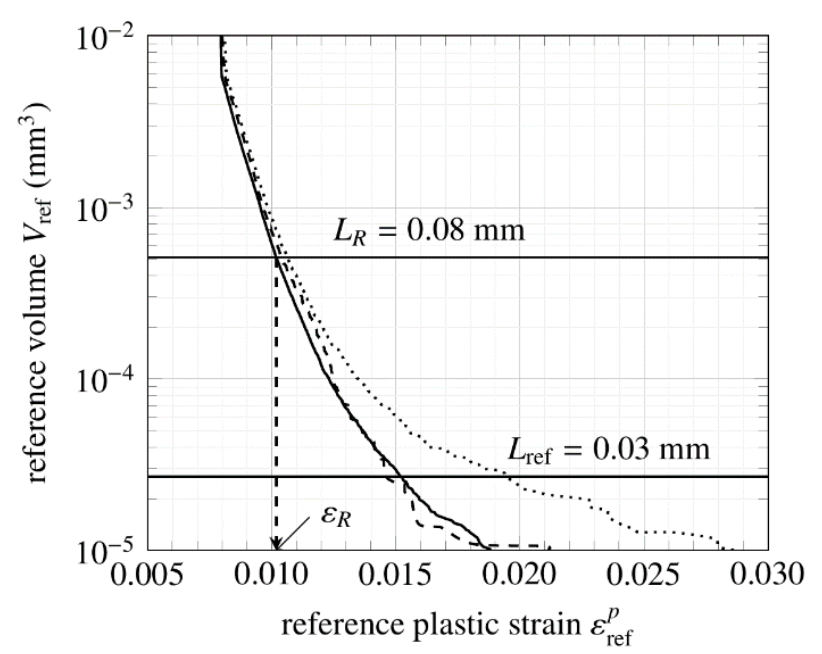

Fig. 12. Reference volume $V_{\text {ref }}$ vs. reference plastic strain $\varepsilon_{\text {ref }}^{P}$ obtained from different meshes with $83128(--)$, $113936(\cdots)$, and 254637 (-) elements.

\section{Application of the representative- volume method to ex-service pits}

Finally, the proposed procedure is used to estimate the crack initiation life of six ex-service pits located at the firtree of a real HPT disc taken from an operated aero engine. Since the artificial pit population in Section 2 was designed to use similar loads and pit sizes as these exservice pits, it is now possible to use the estimator (11) based on Fig. 10b also for ex-service pit life estimation. This means, that only the calculation of the representative strain of the ex-service pits is necessary.

According to Section 3, the surfaces of the exservice pits have been scanned and smoothly approximated by a B-spline surface. They were then implemented in a finite element model to obtain the stress field and plastic strains. In the present case, the simulation load was set to $100 \%$ of the nominal load. Once the finite element problem is solved, a volumetric accumulation diagram similar to Fig. 8 may be drawn for each exservice pit. The representative plastic strain $\varepsilon_{R, k}$ of each ex-service pit $k$ is computed by using the optimum reference length $L_{R}$ found in Section 4, and it is used as input $q_{k}=\varepsilon_{R, k}$ for the estimator (11) to obtain the associated crack initiation lives $N_{\mathrm{I}, k}, k=1, \ldots, 6$.

To compare the estimated crack initiation lives of ex-service pits with those of artificial pits, we consider the artificial pit data set at the optimum reference length, 
Fig. 10b, and transfer it to a traditional fatigue life diagram in Fig.13, i.e., a non-logarithmic drawing with representative strain $\varepsilon_{R}$ as ordinate and cycles to crack initiation $N_{I}$ as abscissa. It is observed that the ex-service pits (grey markers) have a higher representative strain than most of the artificial pits. Therefore, a rather short crack initiation life may be expected from the estimating curve.

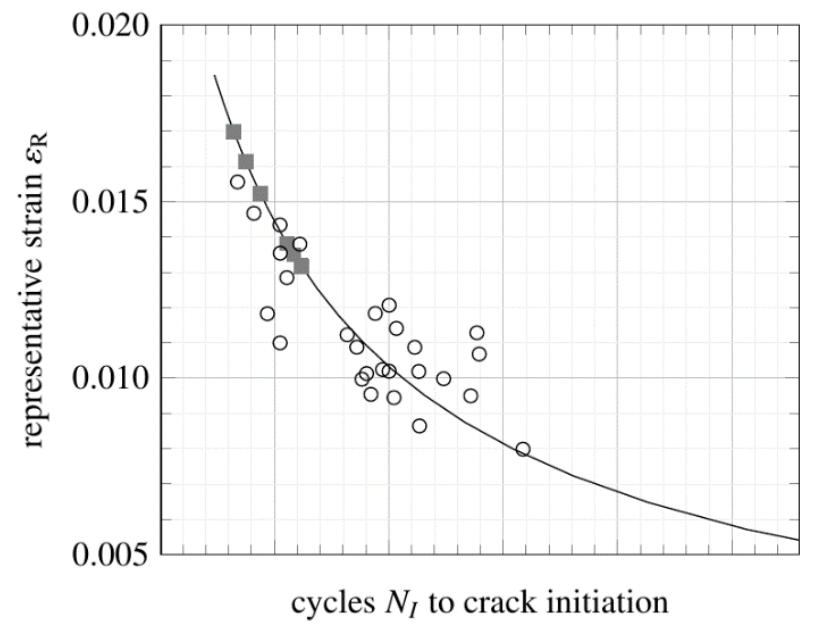

Fig. 13. Crack initiation life for ex-service pits ( $\square$ ), determined with $L_{\text {ref }}=0.08 \mathrm{~mm}$ ) and artificial pits (०).

\section{Conclusions}

Classical crack initiation predictors such as pit depth seem to offer only a low correlation with crack initiation life and are highly dependent on pit shape, as a test campaign on 28 artificially produced pits shows. Therefore, a new strategy called the representative-volume method is developed, which overcomes this limitation by formulating the representative strain as a predictor. The crack initiation life may be estimated by an exponential function trained by a population of 28 artificially pitted test specimens. The estimator is rather robust against different pit sizes and shapes, loading, and meshing of the finite element analysis. The proposed approach provides a valuable tool for corrosion pit characterization, which significantly reduces the conservatism of current methods and provides a platform for future technology development based on robotic visual inspection, automated damage measurement, and decision on damage repair or acceptance without human involvement.

This work has been carried out in collaboration with Rolls-Royce Deutschland. The first author is funded by ANTriebE (Adaptative Neuorientierung von TriebwerksEntwurfssystemen) financed by the State of Brandenburg and the European Regional Development Fund. Rolls-Royce Deutschland's permission to publish this work is greatly acknowledged. The authors also thank the National Physical Laboratory for their assistance in the design, production and analysis of artificial corrosion pits.

\section{References}

1. B. M. Schönbauer, S. E. Stanzl-Tschegg, A. Perlega, R. N. Salzman, N. F. Rieger, S. Zhou, A. Turnbull, and D. Gandy, Int. J. Fatigue 65, 33 (2014).

2. B. M. Schönbauer, A. Perlega, U. P. Karr, D. Gandy, and S. E. Stanzl-Tschegg, Int. J. Fatigue 76, 19 (2015).

3. S. Xu and Y. Wang, Int. J. Fatigue 55, 193 (2014).

4. S. I. Rokhlin, J. Y. Kim, H. Nagy, and B. Zoofan, Eng. Fract. Mech. 62, 425 (1999).

5. A. Shekhter, B. R. Crawford, C. Loader, and W. Hu, Eng. Fail. Anal. 55, 193 (2015).

6. D. L. McDowell, Mater. Sci. Eng. A 468-470, 4 (2007).

7. P. Shi and S. Mahadevan, Eng. Fract. Mech. 68, 1493 (2001).

8. M. Mao, X. Zhang, S. Tu, and F. Xuan, J. Aircr. 51, 805 (2014)

9. M. Cerit, K. Genel, and S. Eksi, Eng. Fail. Anal. 16, 2467 (2009).

10. A. Turnbull, L. N. McCartney, and S. Zhou, Scr. Mater. 54, 575 (2006).

11. M. Amiri, A. Arcari, L. Airoldi, M. Naderi, and N. Iyyer, Corros. Sci. 98, 678 (2015).

12. P. Hu, Q. Meng, W. Hu, F. Shen, Z. Zhan, and L. Sun, Corros. Sci. 113, 78 (2016).

13. M. D. Sangid, Int. J. Fatigue 57, 58 (2013).

14. J. T. Burns, J. M. Larsen, and R. P. Gangloff, Fatigue Fract. Eng. Mater. Struct. 34, 745 (2011).

15. B. Erice, F. Gálvez, D. A. Cendón, and V. SánchezGálvez, Eng. Fract. Mech. 79, 1 (2012).

16. A. Turnbull, L. Wright, and L. Crocker, Corros. Sci. 52, 1492 (2010). 